\title{
Role of Dental Stem Cells in Regenerative Medicine
}

\author{
Dr. Nima ${ }^{1}$, Dr. Mangala P.R ${ }^{2}$, Dr. Veena S. $N^{3}$, Dr. Krupashankar $\mathbf{R}^{4}$, Dr. Kavitha A $\mathbf{P}^{5}$ \\ ${ }^{1}$ Post Graduate Student, Department of Oral medicine and Radiology, Coorg Institute of Dental sciences, Virajpet, Karntaka \\ ${ }^{2}$ Principal \& H.O.D, Department of Oral medicine \& Radiology, Daswani Dental college, Ranpur, Kota, Rajasthan \\ ${ }^{3}$ Proffesor and Head, Department of Oral medicine and Radiology, Coorg Institute of Dental sciences, Virajpet, Karntaka \\ ${ }^{4}$ Reader, Department of Oral medicine and Radiology, Coorg Institute of Dental sciences, Virajpet, Karntaka \\ ${ }^{5}$ Senior lecturer, Department of Oral medicine and Radiology, Coorg Institute of Dental sciences, Virajpet, Karntaka
}

\begin{abstract}
Stem cell therapy has a promising future for tissue engineering and regenerative medicine. However stem cell technology is still in its infancy, interdisciplinary co-operation is needed to achieve successful clinical applications. Dental stem cells have drawn attention in recent years because of their accessibility, plasticity and high proliferative ability. Several types of dental stem cells have been identified, which include those derived from adult human dental pulp, primary exfoliated deciduous teeth, periodontal ligament, dental follicle and permanent third molars. Similar to mesenchymal stem cells, they also undergo self renewal and have multipotent differentiation ability, but do not have the ethical issues associated with the other sources of stem cells. This review describes about the potential of different types of dental stem cells.
\end{abstract}

Keywords: Dental stem cells, Regenerative medicine, DPSC's, SHED cells, PDLSC's, DFSC's, SCAP cells, Tissue Engineering

\section{Introduction}

Stem cell therapy, an emerging novel strategy for therapeutics has made immense contribution to the field of Regenerative medicine and Tissue engineering which has cured many of the incurable diseases and has generated new tissues and organs ${ }^{1}$. The artificial prosthesis like implants and crowns will soon be replaced with stem cell induced natural organ and tissue parts including human tooth. There are possibilities for regenerating the diseased, injured or traumatized oral mucosal tissues using dental stem cells. Regenerative medicine is defined as an emerging interdisciplinary field of research and clinical applications focused on the repair, replacement or regeneration of cells, tissues or organs to restore impaired function resulting from any cause, including congenital defects, disease, trauma and ageing $^{2}$. The term Tissue Engineering was first coined by Y.C Fung, a pioneer in the field of biomechanics and bioengineering in 1985 which is in fact a part of regenerative medicine ${ }^{1}$.Tissue engineering is defined as an interdisciplinary field that applies the principles of engineering and life sciences, towards the development of biological substitutes that restore, maintain or improve tissue function ( Langer and Vacanti,1993) ${ }^{3}$. In the last decade, dentistry has begun to explore the potential application of stem cells and tissue engineering towards the repair and regeneration of dental structures. This conceptual approach to therapy, named "REGENERATIVE DENTISTRY" will have its place in the clinical practice of dentistry in the future. This review brings to light the fundamental concepts of various types of dental stem cells, its source and its potential implications within the context of regenerative dentistry ${ }^{1}$

\section{Stem Cells}

Stem cells are unspecialized cells that can differentiate and reproduce themselves into various cell types ${ }^{4}$. Properties of self renewal and differentiation into specialized cells make them unique ${ }^{5}$.

\section{Classification of Stem Cells ${ }^{6}$}

1. On the basis of Source

a. Autologous: Obtained from the same individual to whom they are to be implanted.

b. Allogenic: Obtained from the donor of the same species.

c. Xenogenic: Obtained from the donor of another species.

d. Syngenic/Isogenic: Obtained from genetically identical organisms.

2. On the basis of Potency

a. Totipotent: differentiate into all embryonic and extra-embryonic cell types.

b. Pluripotent: differentiate into all types of cells, other than the cells of the embryonic membrane.

c. Multipotent: differentiate into more than one mature cell.

d. Unipotent: differentiate into only one type of cells.

3. On the basis of origin: 
International Journal of Science and Research (IJSR)

ISSN (Online): 2319-7064

Index Copernicus Value (2013): 6.14 | Impact Factor (2014): 5.611

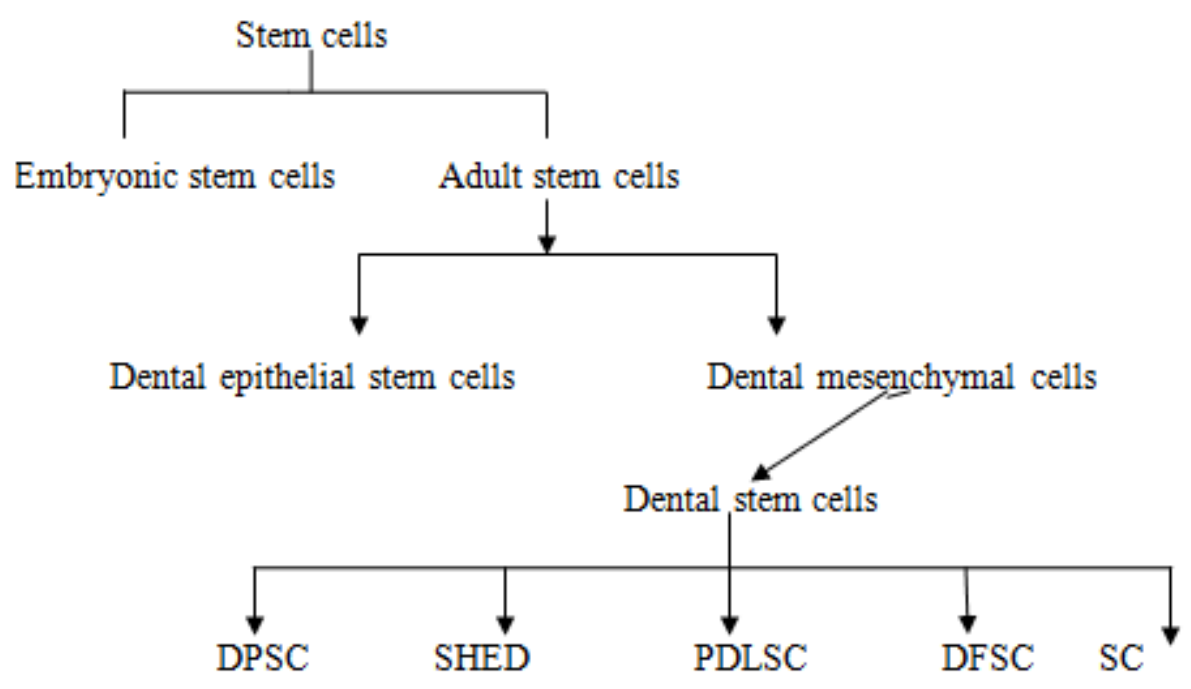

( Gronthos et al) (Miura et al, 2003) (Seo et al,) (Sonoyama et al 2006)

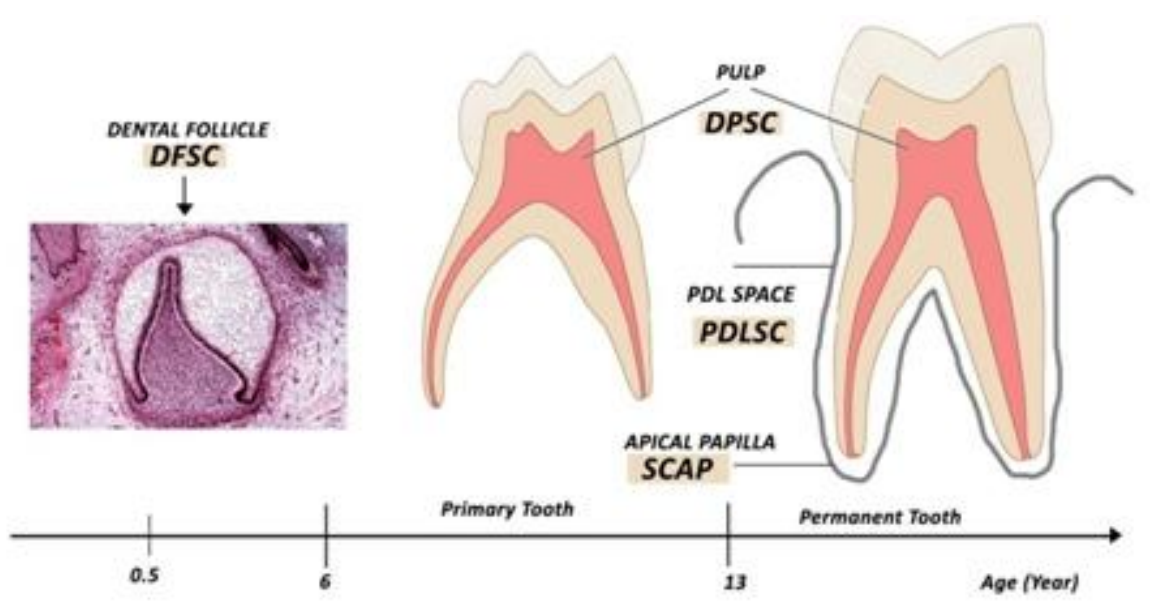

Figure 1: Types of Dental Stem cells

\section{Dental Pulp Stem Cells}

DPSC'S were the first type of dental stem cells to be isolated from the human permanent third molars by Gronthos et al in $2000^{6}$. They exhibited high proliferation rate and high frequency of colony formation that produced calcific nodules. Bakopoulou A et al, reported that DPSC cultures from impacted $3^{\text {rd }}$ molars can differentiate into odontoblast like cells which has increased mineralization and dentin forming potential invitro ${ }^{7}$. Dental pulp is a store house of a high percentage of stem cells, which can differentiate into bone, dentin, muscle and nerve tissue. DPSC's can be easily retrieved from the freshly extracted permanent tooth without any associated morbidities ${ }^{6}$. The compatability and attachment to various biomaterials is high for DPSC's ${ }^{8}$. Kerkis et al described about a sub population of DPSC's named as immature dental pulp stem cells (IDPSC's). DPSC's have a longer survival time because these cells are quiescent (non dividing), in perivascular environment where their stem cell characteristics are maintained for a longer duration of time. These cells become activated whenever there is a need for more cells as in tissue injury. A process where new dentin matrix is laid down at the injured site for the protection of the injured pulp is known as "Reparative dentinogenesis" is an example showing the potential of DPSC's to proliferate and produce odontoblasts following a dental injury. Their property of differentiating into functionally active neurons used for treating various neuronal disorders was suggested by Arthur et al 9 . When recombined with bio-degradable scaffolds, DPSC's form tissues like dentin \& pulp with irregular shape in vitro. The immunosuppressive activity of DPSC's can be advantageous in cases of stem cell transplantation ${ }^{6}$. DPSC's can also help in cardiac repair after myocardial infarction. Jamal et al reported that, the left coronary artery was ligated in nude rats and DPSC's were transplanted to the border of the infarction zone. Evidence of cardiac repair was noted by improved cardiac function, increase in the number of vessels and reduction in the infarct size after 4 weeks of transplantation'. DPSC's can regenerate dentin and provide it with oxygen, nutrition and innervations, whereas the hard dentin can protect the soft dental pulp tissue. Together they maintain the intergrity of tooth shape and function ${ }^{7}$. DPSC's demonstrated the ability to generate functional dental tissue in the form of dentin/pulp like complexes when transplanted in vivo into immuno-compromised mice. . In addition DPSC's can express neural markers and differentiate into functionally active neurons, suggesting their potential in cellular therapy for neuronal disorders ${ }^{7}$. DPSC derived cells integrate into the host brain and may serve as useful sources 


\section{International Journal of Science and Research (IJSR) \\ ISSN (Online): 2319-7064 \\ Index Copernicus Value (2013): 6.14 | Impact Factor (2014): 5.611}

of neuro and gliogenesis in vivo, especially when the brain is injured ${ }^{7}$. Govindasamy et al has shown that the transdifferentiation of DPSC'S into islet cell aggregates, those similar to pancreatic islet cells, released insulin in a glucose dependent manner in vitro which can be used for the treatment of diabetes ${ }^{10}$.

\section{Role in Regenerative Endodontics}

Dental pulp stem cells have established their role in regenerative endodontics. Regenerative endodontics is a biological procedure designed to replace the damaged dentin-pulp complex in a non vital tooth. The pulp chambers and canals of a necrosed tooth is debrided, irrigated and the canal is filled up with dental pulp stem cells, growth factors and molecules like calcium hydroxide \& poly antibiotic pastes. Stem cells and the proteins combine together, prompting the growth of new pulpal cells, blood vessels and nerves. Thus new vital pulp is formed. Regenerative endodontics will soon take over the conventional endodontic restorative procedures.

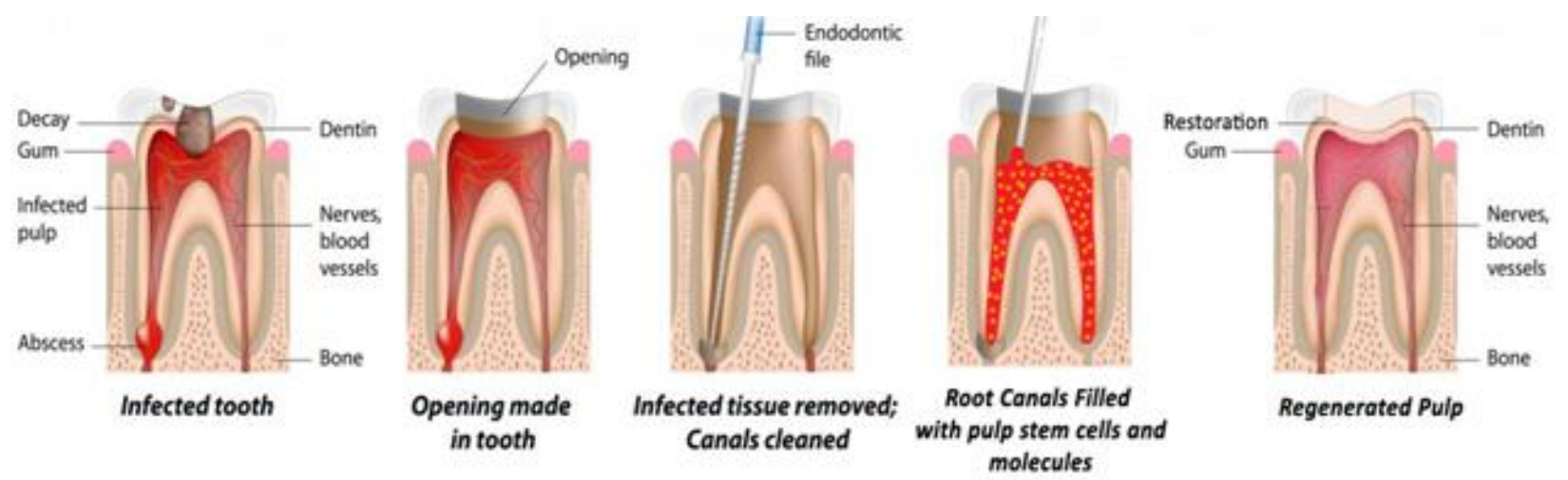

Figure 2: Steps in Regenerative Endodontics

\section{SHED cells (Stem cells from human exfoliated deciduous teeth.)}

Miura et al, in 2003 first isolated stem cells from human exfoliated deciduous teeth ${ }^{11}$. Mesenchymal progenitors have been isolated from the pulp of the human deciduous incisors. These cells were named SHED (stem cells from Human exfoliated deciduous teeth) and they exhibited high plasticity since they could differentiate into neurons, osteoblasts and odontoblasts, adipocytes. SHED cells can induce bone or dentin formation in vivo, but in contrast to DPSC's they failed to produce dentin-pulp complex. Being immature cells as they can differentiate into various cell types. They offer painless stem cell collection because they are retrieved from a tissue that is disposable and easily accessible. According to L.T Friedlander et al they have higher proliferation rate and population doublings ${ }^{6}$.

\section{SCAP cells (Stem cells from the apical papillae)}

Stem cells from the apical papilla are a population of multipotent stem cells isolated from the apical papilla at the root tip of a human tooth. Compared with DPSC, SCAP cells have greater numbers of STRO-1 positive cells, a greater number of population doublings, faster proliferation, and increased capacity for in vivo dentin regeneration. SCAP cells are positive for telomerase activity which is present in the embryonic stem cells and suggests a very immature source of cells available for hard tissue regeneration. According to Miura et al SCAP might be the source of primary odontoblasts involved in the development dentine of the root, in contrast to DPSC, which are likely to be the source of replacement odontoblasts involved in reparative dentin formation ${ }^{6}$. These cells express neurogenic and myogenic markers and are capable of odontoblastic, osteoblastic, differentiation. They have capacity for dentin and pulp regeneration using revascularization and revitalization techniques by inducing bleeding together with the apical papilla cells. SCAP are used for the formation of roots and dentin and also help in the apexogenesis after the disinfection of root canals in children. SCAP can convert themselves into the Dental pulp stem cells. SCAP cells appear to undergo odontogenic differentiation as measured by the expression of the markers dsp, alp, bsp ${ }^{6}$.

\section{Periodontal Ligament Stem Cells (PDLSC'S)}

Periodontal ligament stem cells were isolated and characterised by Seo et al in 2004. In vitro studies demonstrated a longer life span, high proliferation rate and population doublings for PDLSC's. According to Nagatomo et al PDLSC's can differentiate into cementoblast-like cells, adipocytes and collagen forming cells in vitro and has the capacity to regenerate a cementum / PDL like structure in vivo $^{6}$.The concept that stem cells may reside in the periodontal tissues was first proposed almost 20 years ago by Melcher, who queried whether the three cell populations of the periodontium (cementoblasts, alveolar bone cells and periodontal ligament fibroblasts) ultimately derived from a single population of ancestral cells or stem cells ${ }^{12}$. 


\section{International Journal of Science and Research (IJSR) ISSN (Online): 2319-7064}

Index Copernicus Value (2013): 6.14 | Impact Factor (2014): 5.611

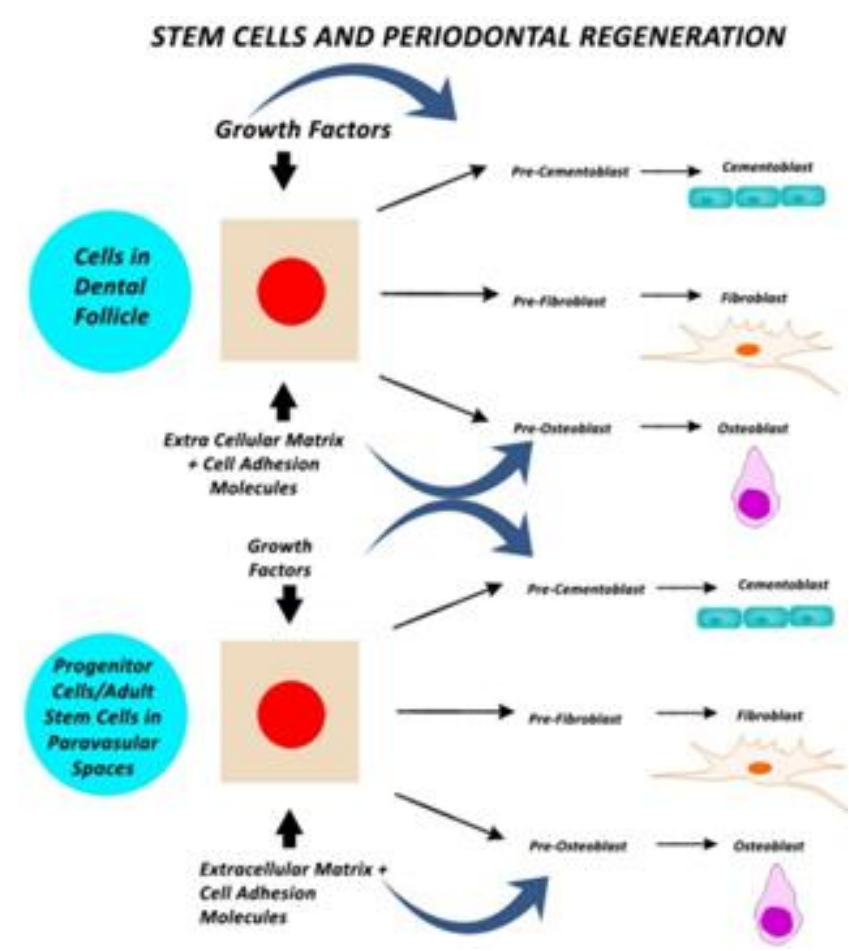

Figure 3: Stem cells in Periodontal Regeneration

PDLSC's retain the stem cell properties and tissue regeneration capacity which can be used to create a biological root. Autologous PDLSC's showed the best regenerating capacity of PDL, alveolar bone and cementum as well as peripheral nerve and blood vessel which were evaluated by conventional and immune histology. Studies reported that PDLSC's could regenerate periodontium, highlighting their potential in the therapy of periodontitis. Fibroblastic activity of PDLS's can be used in the treatment of Oral sub mucous fibrosis and has been found to reduce the facial wrinkles in mice.

\section{Dental Follicular Progenitor Cells (DFSC's)}

The dental follicle is a multipotent tissue and the stem cells isolated from them are named as dental follicle progenitor cells. These were first isolated from the follicle of human impacted $3^{\text {rd }}$ molars. According to Kemoun $\mathrm{P}$ et al, the dental follicular stem cells can differentiate into cementoblasts in vitro (Handa $\mathrm{K}$ et al) and immortalized dental follicular cells are able to re-create a new periodontal ligament (PDL) after in vivo implantation (Yokoi et al) ${ }^{6}$. The dental follicle has long been considered as a multipotent tissue, based on its ability to generate cementum, bone and PDL from the ecto-mesenchyme derived fibrous tissue. DFSC's can induce periodontal and bone regeneration. DFSC form the PDL by differentiating into PDL fibroblasts that secrete collagen and interact with the fibers on the surfaces of the adjacent bone and cementum. These cells isolated from the human $3^{\text {rd }}$ molars are characterised by their rapid attachment in culture, and the ability to form compact calcified nodules in vitro.

\section{Triad of Tissue Engineering}

Reconstitution of bioengineered tooth germ, like that for other organs, is one of the future prospective of stem cells which requires that single cells are completely dissociated from both the epithelium and the mesenchyme, and the correct placement of cells is a major issue. The most successful technique for tissue engineering of simple tissues is the use of biodegradable scaffolds into which the cells are seeded and adopting the shape of the scaffold ${ }^{13}$.

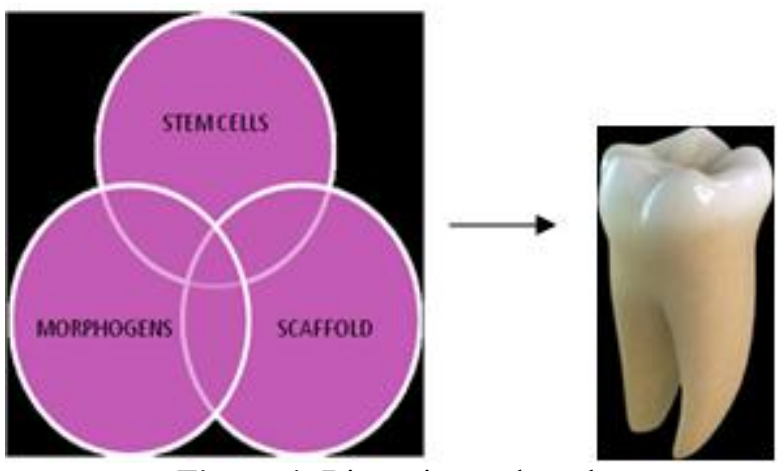

Figure 4: Bioengineered tooth

\section{SCAFFOLDS}

Scaffolds are suitable substrates on which the stem cells can grow in the presence of growth factors. The seeding of cells on tissue engineering scaffolds is known as "creating a tissue construct".

\section{Requirements of a scaffold}

- Effective for the transport of nutrients, oxygen and waste.

- Biocompatible and non-toxic

- Physically and mechanically strong.

- Porous to allow cell placement, distribution and proliferation.

- Permeability of the culture medium.

- Ability for in vivo vascularisation.

- Maintain osteoblastic cell phenotype.

- Ease of fabrication.

- Gradually degraded and replaced by regeneration tissue, retaining the features of the final tissue structure.

Scaffolds can be classified as natural or synthetic. The natural scaffolds are more bio compatible, but the synthetic scaffolds offer improved control over physico-chemical properties of the medium. They are more conducive to the growth of new tissue and relatively contraction free ${ }^{6}$. The scaffold provides a physical, chemical and biological three dimensional micro environment for cell growth and differentiation, promoting cell adhesion and migration. The scaffold serves as a carrier for morphogen in protein therapy and for cells in cell therapy ${ }^{11}$.

\begin{tabular}{|c|c|}
\hline $\begin{array}{c}\text { Natural / Biocompatible } \\
\text { Scaffolds }\end{array}$ & \begin{tabular}{c} 
Synthetic / Artificial Scaffolds \\
\hline Collagen
\end{tabular} \\
\hline Glycosaminoglycans & Poly lactic acid \\
\hline Delyglycolic acid \\
\hline Fibrin & Polyethylene glycol \\
\hline Prp & Arginine \\
\hline Emdogain & Agarose \\
\hline & Chitosan \\
\hline & Hydroxyapatite \\
\hline & Tricalcium phosphate \\
\hline & Polyepsilon \\
\hline & Titanium \\
\hline & Bioceramics \\
\hline & Hydrogel \\
\hline
\end{tabular}




\section{International Journal of Science and Research (IJSR) \\ ISSN (Online): 2319-7064}

Index Copernicus Value (2013): 6.14 | Impact Factor (2014): 5.611

\section{Growth Factors}

Growth factors are protiens that bind to the receptors on the cell and induce cellular proliferation or differentiations. Many growth factors are quite versatile, stimulating cellular division in numerous cell types, while others are more cell specific ${ }^{11}$. Bone Morphogenetic Protiens (BMP) are growth factors required in tooth development and regeneration. Recombinant human proteins, insulin like growth factor-1 together with collagen has been found to induce complete dentin bridging and tubular dentin formation (Lovschall et al, 2001 ).Growth factors may play key roles in signaling many of the events of tertiary dentinogenesis, a response of pulp-dentin repair. FGF-2 plays a key role not only as a differentiation inducing factor in the injury repair process of pulpal tissue, but also as a positive regulator of chemokine expression, which may help in tissue engineering and pulp regeneration using human DPSC's ${ }^{11}$.

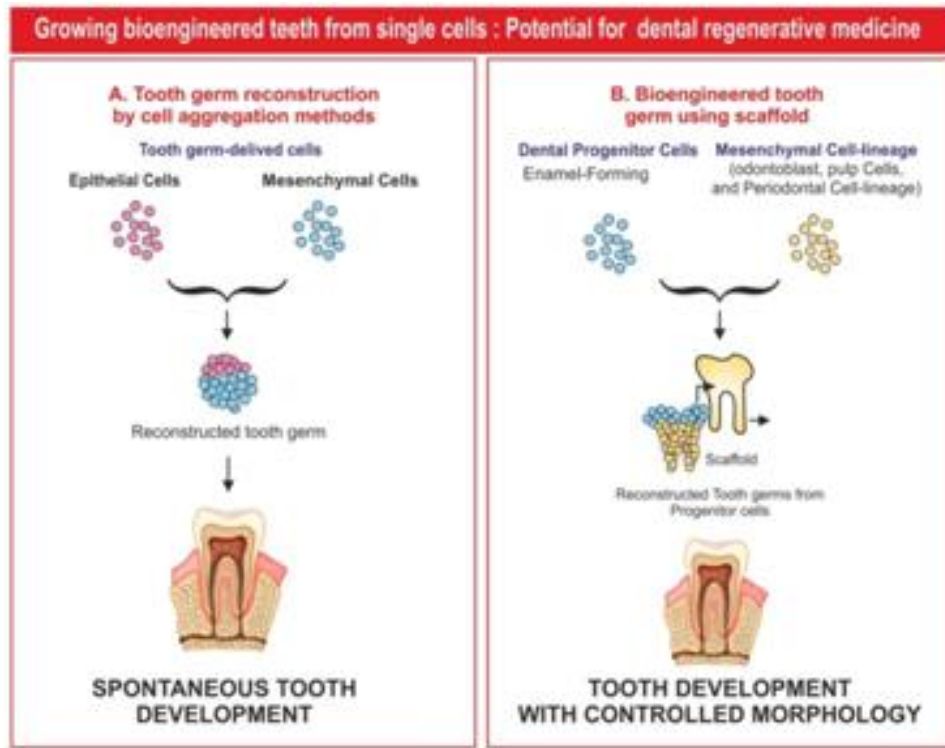

Figure 5: (A) Single epithelial, mesenchymal cells are dissociated from the tooth germ at an early developmental stage. Dissociated cells reaggregate to regenerate bioengineered tooth germ. Regenerated tooth germ spontaneously develops into a tooth. B) Enamel forming progenitor cells and dentin forming progenitor cells are arranged in their proper anatomical relationship on a scaffold. The reconstructed tooth germ from the progenitor cells leads to tooth development with controlled morphology.

\section{Collection, Isolation, and Preservation of Stem Cells ${ }^{14}$}

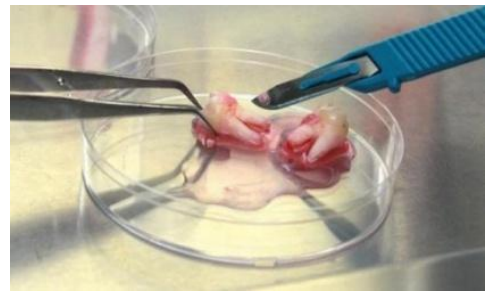

Figure 6: Stem cell collection

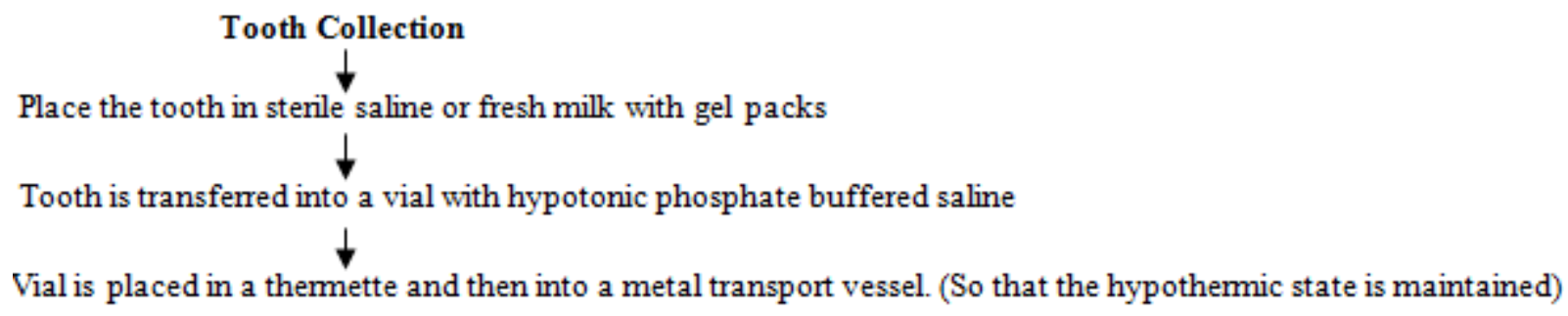

\section{Stem Cell Isolation}




\section{International Journal of Science and Research (IJSR) \\ ISSN (Online): 2319-7064}

Index Copernicus Value (2013): 6.14 | Impact Factor (2014): 5.611

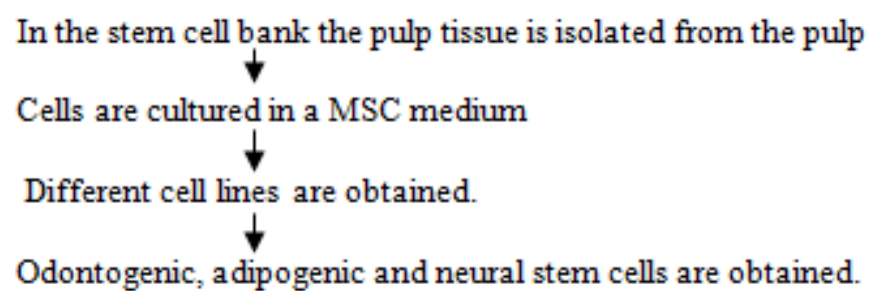

Entire process should not exceed $40 \mathrm{hrs}$

\section{Stem Cell Storage}

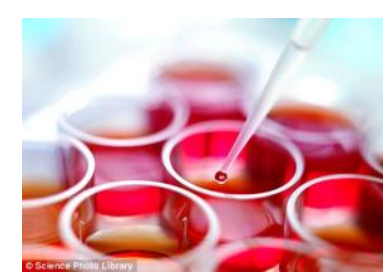

Figure 7: Preservation of Stem cells

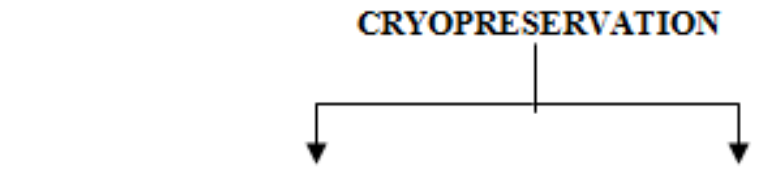

Magnetic freezing (cell live system)

Preserved in liquid nitrogen at $-150^{\circ} \mathrm{C}$

Uses a magnetic field (object chilled below freezing point)

\section{Conclusion}

The role of a $3^{\text {rd }}$ dentition (stem cell induced teeth) to replace the missing natural tooth is an attractive concept ${ }^{13}$. Role and status of adult dental stem cell is still being debated, but in near future its therapeutic outcome will define their importance ${ }^{16}$. Several reports on the characterization and differentiation of dental stem cells have been published and is a growing field of interest ${ }^{17}$. Dental regenerative medicine will soon make its progress in such a way that the organ replacement therapies and teeth replacement with dental stem cells will soon become a reality ${ }^{15}$. The next decade may be an era of dental stem cells where in implants will soon fade out and stem cell based teeth will soon take up its role.

\section{References}

[1] Luciano Casagrande, Mabel M. Cordeir, Silvia A, Nor Jacques E. Dental pulp stem cells in regenerative dentistry. Odontology 2011; 99:1-7 (C) The Society of The Nippon Dental University 2011.

[2] Greenwood HL, Singer PA, Downey GP, Martin DK, Thorsteinsdottir H, Daar AS. Regenerative medicine and the developing world. PLoS Med 2006; 3: e381).

[3] Li Peng, Ling Ye, Xue-dong Zhou Mesenchymal Stem Cells and Tooth Engineering. International Journal of Oral Science. 2009; 1(1): 6-12.

[4] S. Bajada, I. Mazakova, B.A. Ashton. Stem cells for regeneration.In: N Ashammakhi, R Reis, \& F Chiellini, Eds. Topics in Tissue Engineering.Expertissues E book. Vol. 4.

[5] S.S. Sandhu, Meera Nair Stem Cells: Potential Implications for tooth regeneration and tissue engineering in Dental Science. People's Journal of Scientific Research. 2009; Vol.2 (1), Jan.

[6] Rita Chandki, M. Kala , Priyank Banthia , Ruchi Banthia. From Stem to Roots: Tissue engineering in Endodontics Biomaterials and Bioengineering in Dentistry J Clin Exp Dent. 2012;4(1):e66-71

[7] Carlos estrela, Ana Helena ,Gonçalves de alencar , Gregory Thomas kitten, Eneida Franco. Mesenchymal Stem Cells in the Dental Tissues: Perspectives for Tissue Regeneration. Braz Dent J (2011); 22(2): 91-98.

[8] Ranganathan K, Vidya Lakshminarayanan. Stem cells of the dental pulp. Indian Journal of Dental Research. 2012; 23(4).

[9] Muhammad Fawwaz Abdullah, Kannan Thirumulu Ponnuraj, and Khairani Idah Mokhtar. DPSCs and SHED in Tissue Engineering and Regenerative Medicine. The Open Stem Cell Journal. 2013; (4): 1-6.

[10] Govindasamy V, Ronald VS, Abdullah AN, Nathan KR, $\mathrm{Ab}$ Aziz ZA, Abdullah et al. Differentiation of dental pulp stem cells into islet like aggregates. J Dent Res 2011; 90:646-52.

[11]Deepak B.S., Nandini D.B. \& S. Naik. Tissue Engineering: Is it the future of Endodontics .People's Journal of Scientific Research. Jan. 2011; Vol. 4(1),

[12] P.Mark Bartold, Songtao Shi \& Stan Gronthos. Stem cells an periodontal regeneration. Periodontology. 2006; Vol. 40, 164-172.

[13] Rachel Sartaj and Paul Sharpe. Biological tooth replacement .J. Anat. 2006; (209), pp503-509.

[14] S Rai, M Kaur, S Kaur. Applications of stem cells in interdisciplinary Dentistry and beyond - An overview. Annals of Medical and Health Sciences Research.3 (2):245-254. 


\section{International Journal of Science and Research (IJSR) \\ ISSN (Online): 2319-7064}

Index Copernicus Value (2013): 6.14 | Impact Factor (2014): 5.611

[15]Etsuko Ikeda \& Takashi Tsuji .Cell- \& Tissue-based Therapy. Growing b bioengineered teethfrom single cells: potential for dental regenerative medicine .Japan Expert Opin. Biol. Ther.(2008); 8(6).

[16] Rosenzweig A: Cardiac cell therapy- mixed results from mixed cells. N. Engl.J.Med. 2006; (12), 1274-1277.

[17] Bettina Lindroos, Katja Ma enpa, Timo Ylikomi .Characterisation of human dental stem cells and buccal mucosa fibroblasts. Biochemical and Biophysical Research Communications. 2008; 329-335. 